\title{
Transverse Acoustic Modes of Biogenic and $\alpha, \omega$-Polyamines: A Study by Inelastic Neutron Scattering and Raman Spectroscopies Coupled to DFT Calculations
}

\author{
Luís A. E. Batista de Carvalho, ${ }^{\dagger}$ M. Paula M. Marques, ${ }^{*},+, *$ and John Tomkinson ${ }^{\S}$ \\ Research Unit "Molecular Physical-Chemistry”, University of Coimbra, 3000 Coimbra, Portugal, Biochemistry \\ Department, University of Coimbra, 3000 Coimbra, Portugal, and ISIS Facility, The Rutherford Appleton \\ Laboratory, Chilton, United Kingdom
}

Received: July 28, 2006; In Final Form: September 19, 2006

\begin{abstract}
A complete analysis of the transverse acoustic modes (TAMs) for the homologous series of $\alpha, \omega$-diamines $\left(\mathrm{H}_{2} \mathrm{~N}\left(\mathrm{CH}_{2}\right)_{n} \mathrm{NH}_{2}\right)(n=2-10, n=12)$ as well as for the biogenic polyamines spermidine and spermine was undertaken, by Raman and inelastic neutron scattering (INS) spectroscopies combined with density functional theory (DFT) calculations. A complete assignment of the whole set of TAMs was carried out, for both the undeuterated and N-deuterated species. 1,2-Diaminoethane was found to display exceptional behavior, probably due to the formation of dimers in the solid state. An $n$-even $/ n$-odd dependence of the low frequency INS pattern was observed for these polyamines. The very good accordance between their INS experimental TAMs and the ones previously reported for the corresponding $n$-alkanes suggest a close conformational similarity between these systems.
\end{abstract}

\section{Introduction}

Biogenic polyamines, present in almost all living organisms, are products of the decarboxylation of amino acids. Putrescine1,4-diaminobutane, $\mathrm{H}_{2} \mathrm{~N}\left(\mathrm{CH}_{2}\right)_{4} \mathrm{NH}_{2}$-is the first member in the polyamine pathway, biosynthesized from arginine and the precursor of spermidine $\left(\mathrm{H}_{2} \mathrm{~N}\left(\mathrm{CH}_{2}\right)_{3} \mathrm{NH}\left(\mathrm{CH}_{2}\right)_{4} \mathrm{NH}_{2}\right)$ and spermine $\left(\mathrm{H}_{2} \mathrm{~N}\left(\mathrm{CH}_{2}\right)_{3} \mathrm{NH}\left(\mathrm{CH}_{2}\right)_{4} \mathrm{NH}\left(\mathrm{CH}_{2}\right)_{3} \mathrm{NH}_{2}\right)$. Under physiological conditions, these linear amines are totally protonated and behave as natural polycations. They are known to be implicated in a bewildering number of cellular functions, ${ }^{1-6}$ being essential for eukaryotic cell growth and differentiation, as well as for maintaining the native structure of several biological macromolecules while affecting the activity of others, ${ }^{1,7}$ through tightly regulated concentration-dependent processes. The understanding of this vital biological role of polyamines requires a thorough knowledge of their structural preferences, which will hopefully allow an explanation of the biochemical mechanisms through which they act in living organisms.

This conformational behavior can be analyzed through vibrational spectroscopic methods coupled to theoretical calculations. Inelastic neutron scattering (INS) spectroscopy, in particular, is a well suited technique for the study of this kind of highly hydrogenated system, specifically for observing low frequency vibrational modes such as the transverse and longitudinal acoustic modes (TAMs and LAMs, respectively).

The full longitudinal acoustic mode INS spectrum of a linear alkane was first determined for $n$-octadecane, ${ }^{8}$ and the whole set of LAMs were observed and assigned for the 5 to 25 $n$-alkanes, ${ }^{9}$ as well as for the polyamines $\mathrm{H}_{2} \mathrm{~N}\left(\mathrm{CH}_{2}\right)_{n} \mathrm{NH}_{2}(n=$

* To whom correspondence should be addressed. Present address: Biochemistry Department, Faculty of Science and Technology, University of Coimbra, Apartado 3126, 3001-401 Coimbra, Portugal. Phone: +351 239854462. Fax: +351 239826541. E-mail: pmc@ci.uc.pt.

† Research Unit "Molecular Physical-Chemistry", University of Coimbra.

$\$$ Biochemistry Department, University of Coimbra.

$\S$ The Rutherford Appleton Laboratory.
$2-10$ and $n=12$ ), spermidine and spermine. ${ }^{10}$ The transverse vibrational modes of the $n$-alkanes pentane to dodecane were also reported. ${ }^{11}$ These transverse acoustic-like vibrations (TAMs), associated to out-of-plane bending modes of the $-\left(\mathrm{CH}_{2}-\mathrm{CH}_{2}\right)_{n}-$ linear chain, are known to be only partially active in optical spectroscopy (both Raman and infrared) and to give rise to intense features in the INS spectra. They occur in the region below $\sim 250 \mathrm{~cm}^{-1}$, which also comprises the external (i.e., intermolecular) vibrational modes.

The present work is focused on the analysis of these lowlying transverse acoustic vibrational modes for the homologous series of $\alpha, \omega$-diamines $\left(\mathrm{H}_{2} \mathrm{~N}\left(\mathrm{CH}_{2}\right)_{n} \mathrm{NH}_{2}\right)(n=2-10, n=12)$, as well as for the biogenic polyamines spermidine and spermine (Figure 1). Vibrational spectroscopy, both Raman and INS, combined with density functional theory (DFT) calculations was used for studying the undeuterated and N-deuterated species. A complete assignment of the TAMs was carried out, following a previous study of the corresponding longitudinal modes (LAMs). ${ }^{10}$

\section{Experimental Methods}

2.1. INS Spectroscopy. The INS spectra were obtained in the Rutherford Appleton Laboratory (United Kingdom), at the ISIS pulsed neutron source, on the TOSCA spectrometer. This is an indirect geometry time-of-flight, high resolution $((\Delta E / E)$ $\sim 2 \%$ ), broad range spectrometer. ${ }^{12}$ Solid compounds $(2-3 \mathrm{~g})$ were wrapped in aluminum foil, while the liquids were placed in thin walled aluminum cans, which filled the beam. The samples were cooled to $\sim 20 \mathrm{~K}$ before collecting the spectra. Data were recorded in the energy range from 16 to $4000 \mathrm{~cm}^{-1}$ and converted to the conventional scattering law, $S(Q, v)$, versus energy tranfer (in $\mathrm{cm}^{-1}$ ) through standard programs.

2.2. Raman Spectroscopy. The Raman spectra performed at room temperature were obtained on a Spex Ramalog 1403 double spectrometer (focal distance $0.85 \mathrm{~m}$, aperture $f / 7.8$ ) equipped with holographic gratings of 1800 grooves $\cdot \mathrm{mm}^{-1}$ and 
A

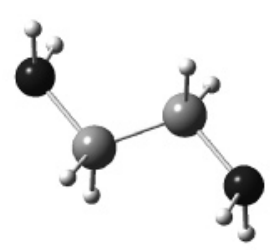

B

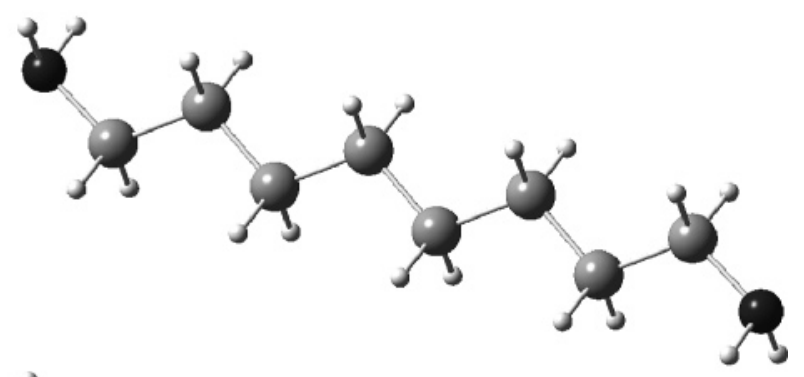

C

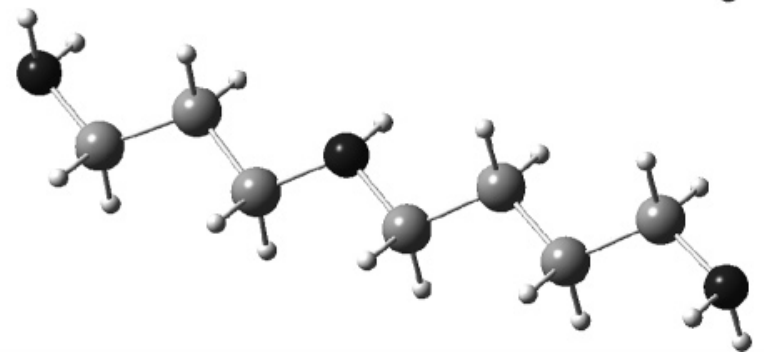

Figure 1. Calculated (B3LYP/6-31G*) all-trans conformations for some of the polyamines studied in this work: (A) 1,2-diaminoethane; (B) 1,8-diaminooctane; (C) spermidine.

a detector assembly containing a thermoelectrically cooled Hamamatsu R928 photomultiplier tube. The spectrometer operated with slits of $320 \mu \mathrm{m}$ and $1 \mathrm{~cm}^{-1} \mathrm{~s}^{-1}$. The $514.5 \mathrm{~nm}$ line of an $\mathrm{Ar}^{+}$laser (Coherent, model Innova 300) was used as excitation radiation, providing $\sim 100 \mathrm{~mW}$ at the sample position.

Below room temperature $(\sim 220 \mathrm{~K})$, a homemade HarneyMiller-type assembly was used, on a triple monochromator Jobin-Yvon T64000 Raman system (focal distance $0.640 \mathrm{~m}$, aperture f/7.5) equipped with holographic gratings of 1800 grooves $\cdot \mathrm{mm}^{-1}$. The premonochromator stage was used in the subtractive mode. The detection system was a liquid nitrogen cooled nonintensified $578 \times 385$ pixel $\left(1 / 2^{\prime \prime}\right)$ charge coupled device (CCD) chip. An $\mathrm{Ar}^{+}$laser (Coherent, model Innova 30005) was used as the light source, the output of which at 514.5 $\mathrm{nm}$ was adjusted to provide between 60 and $80 \mathrm{~mW}$ at the sample position. A $90^{\circ}$ geometry, between the incident radiation and the collecting system, was employed. The entrance slit was set to $200 \mu \mathrm{m}$, and the slit between the premonochromator and the spectrograph was opened to $12 \mathrm{~mm}$.

Samples were sealed in Kimax glass capillary tubes of 0.8 $\mathrm{mm}$ inner diameter. Under the above-mentioned conditions, the error in wavenumbers was estimated to be within $1 \mathrm{~cm}^{-1}$.

2.3. DFT Calculations. The quantum mechanical calculationsgeometry optimization and calculation of the harmonic vibrational frequencies - were performed using the GAUSSIAN 98W program, ${ }^{13}$ within the density functional theory (DFT) approach in order to properly represent the electron correlation effects (particularly important in this kind of nitrogen-containing system). The B3LYP hybrid method, which includes a mixture of HF and DFT exchange terms and the gradient-corrected correlation functional of Lee, Yang, and Parr ${ }^{14,15}$ as proposed and parametrized by Becke, ${ }^{16,17}$ was used, along with the double- $\zeta$ split valence basis set $6-31 \mathrm{G}^{*}{ }^{18}$
Only the geometries with all skeletal dihedral angles equal to $180^{\circ}$ (all-trans) were considered in the present work. Molecular geometries were fully optimized by the Berny algorithm, using redundant internal coordinates: ${ }^{19}$ the bond lengths to within $\sim 0.1 \mathrm{pm}$ and the bond angles to within $\sim 0.1^{\circ}$. The final root-mean-square (rms) gradients were always less than $3 \times 10^{-4}$ hartree $\cdot b^{-1}$ or hartree $\cdot b{ }^{-1} r^{-1}$.

The calculated INS transition intensities were obtained using the a-CLIMAX program, ${ }^{20}$ which calculates the INS spectrum corresponding to the results yielded by the quantum mechanical calculations. In this case, the vibrational mean square atomic displacements are the most important outputs.

2.4. Reagents. All of the polyamines were purchased from Sigma-Aldrich. The N-deuterated compounds were obtained by mixing the amines with $\mathrm{D}_{2} \mathrm{O}(\sim 10 \%$ excess $)$ and distilling under vacuum (with this process being repeated at least three times). The solid amines were purified by sublimation, while the liquids were distilled under vacuum. With all of the samples being air or moisture sensitive, they were always handled in a glovebox under an argon atmosphere.

\section{Results and Discussion}

The conformational preferences of alkylamines are mainly determined by intra- versus intermolecular $(\mathrm{N}) \mathrm{H} \cdots: \mathrm{N}$ and (C) $\mathrm{H} \cdots: \mathrm{N}$ hydrogen bonds, as previously confirmed by both quantum mechanical calculations and vibrational spectroscopy studies. ${ }^{21-23}$ These molecules can adopt different geometries, by varying the dihedral angles that determine their overall orientation, the most common conformations having skeletal dihedral angles near $60^{\circ}$ (gauche), $180^{\circ}$ (trans), and $-60^{\circ}$ (gauche'). However, the crystal packing of the polyamines in the solid state-which corresponds to the present study-is compatible only to the all-trans conformers (Figure 1) that can be compared to saturated linear alkanes, for which the all-trans geometry has long been recognized as being energetically the most favored. ${ }^{24}$ Under these conditions, the formation of intramolecular hydrogen bonds is hindered, and only three effects are relevant in determining conformation: electrostatic and steric forces and intermolecular $\mathrm{R}-\mathrm{N}-\mathrm{H} \cdots \mathrm{NH}_{2}-\mathrm{R}$ interactions (namely, at the terminal $\mathrm{NH}_{2}$ groups of the chain ${ }^{25}$ ), which are probably quite strong due to the linear geometry of the samples and the marked directional nature of these hydrogen bonds. ${ }^{26-29}$ In fact, these hydrogen-type bonds are clearly evidenced by the shift to low frequencies observed for the Raman $v_{\mathrm{NH}_{2}}$ bands when going from the pure liquid to the solid state.

DFT calculations were carried out for both the undeuterated and $\mathrm{N}$-deuterated all-trans configurations of the series of amines $\left(\mathrm{H}_{2} \mathrm{~N}\left(\mathrm{CH}_{2}\right)_{n} \mathrm{NH}_{2}\right)(n=2-10, n=12)$, spermidine and spermine, in view of allowing a complete assignment of their low frequency vibrational spectra. No polarization functions were included in the hydrogen atoms, since it was verified, in previous studies on these systems, ${ }^{10}$ that the $6-31 G^{*}$ basis set yields reliable results (in good accordance with the corresponding MP2/ $6-31 \mathrm{G}^{* *}$ data). The members of the $\mathrm{H}_{2} \mathrm{~N}\left(\mathrm{CH}_{2}\right)_{n} \mathrm{NH}_{2}(n=2-10$, $n=12$ ) series exhibit $C_{2 h}$ symmetry for $n=$ even and $C_{2 v}$ symmetry for $n=$ odd. Spermidine and spermine, in turn, were found to have a much lower symmetry, $C_{1}$ and $C_{2}$, respectively.

A complete assignment of the TAMs for the whole series of polyamines under study is comprised in Tables 1 (undeuterated amines) and 2 (N-deuterated amines). This was carried out in light of the DFT calculations performed for these systems and by comparison with analogous data obtained for the corresponding $n$-alkanes. ${ }^{11}$ In accordance with the LAMs previously reported for the same amines, ${ }^{10}$ all of the TAMs display the 
TAMs of Biogenic and $\alpha, \omega$-Polyamines

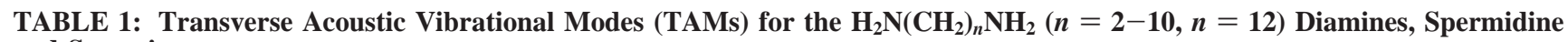
and Spermine

\begin{tabular}{|c|c|c|c|c|c|c|c|c|c|c|c|c|c|}
\hline \multirow[b]{2}{*}{$N$} & & & \multicolumn{11}{|c|}{$i$} \\
\hline & & & 1 & 2 & 3 & 4 & 5 & 6 & 7 & 8 & 9 & 10 & 11 \\
\hline \multirow[t]{3}{*}{4} & 1,2dae $\left(C_{2 h}\right)$ & $\begin{array}{l}\text { exptl (INS) } \\
\text { exptl (Ram) }\end{array}$ & 207 & & & & & & & & & & \\
\hline & & calcd $^{a}$ & $145\left(\mathrm{~A}_{\mathrm{u}}\right)$ & & & & & & & & & & \\
\hline & & $\kappa$ & 0.500 & & & & & & & & & & \\
\hline \multirow[t]{6}{*}{5} & 1,3 dap $\left(C_{2 v}\right)$ & exptl (INS) & 191 & 180 & & & & & & & & & \\
\hline & & & & 174 & & & & & & & & & \\
\hline & & $\mathrm{av}^{b}$ & & 178 & & & & & & & & & \\
\hline & & exptl (Ram) & & 180 & & & & & & & & & \\
\hline & & calcd & $125\left(\mathrm{~A}_{2}\right)$ & $120\left(B_{1}\right)$ & & & & & & & & & \\
\hline & & $\kappa$ & 0.333 & 0.666 & & & & & & & & & \\
\hline \multirow[t]{6}{*}{6} & $1,4 \mathrm{dab}\left(C_{2 h}\right)$ & exptl (INS) & 154 & 199 & 112 & & & & & & & & \\
\hline & 1, & & 161 & 191 & & & & & & & & & \\
\hline & & $\mathrm{av}^{b}$ & 158 & 195 & & & & & & & & & \\
\hline & & exptl (Ram) & 157 & 198 & & & & & & & & & \\
\hline & & calcd & $103\left(\mathrm{~A}_{\mathrm{u}}\right)$ & $164\left(\mathrm{~B}_{\mathrm{g}}\right)$ & $79\left(\mathrm{~A}_{\mathrm{u}}\right)$ & & & & & & & & \\
\hline & & $\kappa$ & 0.250 & 0.500 & 0.750 & & & & & & & & \\
\hline \multirow[t]{6}{*}{7} & $1,5 \operatorname{dap}\left(C_{2 v}\right)$ & exptl (INS) & 134 & 202 & 167 & 111 & & & & & & & \\
\hline & & & & 189 & & 103 & & & & & & & \\
\hline & & $\mathrm{av}^{b}$ & & 196 & & 107 & & & & & & & \\
\hline & & exptl (Ram) & & 183 & 177 & 108 & & & & & & & \\
\hline & & calcd & $89\left(\mathrm{~A}_{2}\right)$ & $162\left(\mathrm{~B}_{1}\right)$ & $150\left(\mathrm{~A}_{2}\right)$ & $68\left(\mathrm{~B}_{1}\right)$ & & & & & & & \\
\hline & & $\kappa$ & 0.200 & 0.400 & 0.600 & 0.800 & & & & & & & \\
\hline \multirow[t]{6}{*}{8} & 1,6 dah $\left(C_{2 h}\right)$ & exptl (INS) & 117 & 177 & 205 & 151 & 99 & & & & & & \\
\hline & & & 109 & 162 & 194 & & & & & & & & \\
\hline & & $a v^{b}$ & 113 & 170 & 200 & & & & & & & & \\
\hline & & exptl (Ram) & & & & 149 & & & & & & & \\
\hline & & calcd & $70\left(\mathrm{~A}_{\mathrm{u}}\right)$ & $141\left(\mathrm{~B}_{\mathrm{g}}\right)$ & $174\left(\mathrm{~A}_{\mathrm{u}}\right)$ & $119\left(\mathrm{~B}_{\mathrm{g}}\right)$ & $50\left(\mathrm{~A}_{\mathrm{u}}\right)$ & & & & & & \\
\hline & & $\kappa$ & 0.167 & 0.333 & 0.500 & 0.667 & 0.833 & & & & & & \\
\hline \multirow[t]{4}{*}{9} & $1,7 \mathrm{dah}\left(C_{2 v}\right)$ & $\begin{array}{l}\text { exptl (INS) } \\
\mathrm{av}^{b}\end{array}$ & 137 & 164 & 199 & 187 & 152 & 119 & & & & & \\
\hline & & exptl (Ram) & & & & & 148 & & & & & & \\
\hline & & calcd & $73\left(\mathrm{~A}_{2}\right)$ & $129\left(\mathrm{~B}_{1}\right)$ & $173\left(\mathrm{~A}_{2}\right)$ & $163\left(\mathrm{~B}_{1}\right)$ & $103\left(\mathrm{~A}_{2}\right)$ & $46\left(B_{1}\right)$ & & & & & \\
\hline & & $\kappa$ & 0.143 & 0.286 & 0.429 & 0.571 & 0.714 & 0.857 & & & & & \\
\hline \multirow[t]{10}{*}{10} & 1,8 dao $\left(C_{2 h}\right)$ & exptl (INS) & 113 & 163 & 188 & 207 & 175 & 141 & 104 & & & & \\
\hline & & & & & 202 & 202 & & & & & & & \\
\hline & & $\mathrm{av}^{b}$ & & & 195 & 205 & & & & & & & \\
\hline & & exptl (Ram) & 118 & & & & & 137 & & & & & \\
\hline & & calcd & $58\left(\mathrm{~A}_{\mathrm{u}}\right)$ & $113\left(\mathrm{~B}_{\mathrm{g}}\right)$ & $159\left(\mathrm{~A}_{\mathrm{u}}\right)$ & $171\left(\mathrm{~B}_{\mathrm{g}}\right)$ & $138\left(\mathrm{~A}_{\mathrm{u}}\right)$ & $82\left(B_{g}\right)$ & $33\left(\mathrm{~A}_{\mathrm{u}}\right)$ & & & & \\
\hline & & $\kappa$ & 0.125 & 0.250 & 0.375 & 0.500 & 0.625 & 0.750 & 0.875 & & & & \\
\hline & $\operatorname{spd}\left(C_{1}\right)$ & $\begin{array}{l}\text { exptl (INS) } \\
\mathrm{av}^{b}\end{array}$ & & 166 & 198 & 211 & 183 & 146 & 110 & & & & \\
\hline & & exptl (Ram) & 131 & & 197 & & 181 & 149 & 111 & & & & \\
\hline & & calcd & 66 & 116 & 165 & 179 & 138 & 85 & 35 & & & & \\
\hline & & $\kappa$ & 0.125 & 0.250 & 0.375 & 0.500 & 0.625 & 0.750 & 0.875 & & & & \\
\hline \multirow[t]{4}{*}{11} & 1,9dan $\left(C_{2 v}\right)$ & $\begin{array}{l}\text { exptl (INS) } \\
\mathrm{av}^{b}\end{array}$ & 123 & 156 & 182 & 204 & 198 & 169 & 136 & 106 & & & \\
\hline & & exptl (Ram) & 123 & & & 203 & & & 140 & & & & \\
\hline & & calcd & $63\left(\mathrm{~A}_{2}\right)$ & $108\left(\mathrm{~B}_{1}\right)$ & $149\left(\mathrm{~A}_{2}\right)$ & $177\left(\mathrm{~B}_{1}\right)$ & $170\left(\mathrm{~A}_{2}\right)$ & $126\left(\mathrm{~B}_{1}\right)$ & $76\left(\mathrm{~A}_{2}\right)$ & $33\left(\mathrm{~B}_{1}\right)$ & & & \\
\hline & & $\kappa$ & 0.111 & 0.222 & 0.333 & 0.444 & 0.556 & 0.667 & 0.778 & 0.889 & & & \\
\hline \multirow[t]{4}{*}{12} & $1,10 \mathrm{dad}\left(C_{2 h}\right)$ & $\begin{array}{l}\text { exptl (INS) } \\
\mathrm{av}^{b}\end{array}$ & 123 & 148 & 170 & 201 & 206 & 180 & 157 & 137 & 107 & & \\
\hline & & exptl (Ram) & & & & 201 & & 176 & & 132 & & & \\
\hline & & calcd & $56\left(\mathrm{~A}_{\mathrm{u}}\right)$ & $98\left(\mathrm{~B}_{\mathrm{g}}\right)$ & $139\left(\mathrm{~A}_{\mathrm{u}}\right)$ & $173\left(\mathrm{~B}_{\mathrm{g}}\right)$ & $180\left(\mathrm{~A}_{\mathrm{u}}\right)$ & $153\left(\mathrm{~B}_{\mathrm{g}}\right)$ & $109\left(\mathrm{~A}_{\mathrm{u}}\right)$ & $63\left(\mathrm{~B}_{\mathrm{g}}\right)$ & $28\left(\mathrm{~A}_{\mathrm{u}}\right)$ & & \\
\hline & & $\kappa$ & 0.100 & 0.200 & 0.300 & 0.400 & 0.500 & 0.600 & 0.700 & 0.800 & 0.900 & & \\
\hline 14 & $1,12 \mathrm{dad}\left(C_{2 h}\right)$ & exptl (INS) & 106 & 132 & 152 & 181 & 197 & 205 & 189 & 156 & 138 & 106 & 87 \\
\hline & & & & & 148 & & 205 & 197 & & 152 & & & \\
\hline & & $a v^{b}$ & & & 150 & & 201 & 201 & & 154 & & & \\
\hline & & exptl (Ram) & & 127 & & 175 & & 197 & & 152 & & 109 & \\
\hline & & calcd & $43\left(\mathrm{~A}_{\mathrm{u}}\right)$ & $80\left(\mathrm{~B}_{\mathrm{g}}\right)$ & $117\left(\mathrm{~A}_{\mathrm{u}}\right)$ & $149\left(\mathrm{~B}_{\mathrm{g}}\right)$ & $172\left(\mathrm{~A}_{\mathrm{u}}\right)$ & $172\left(\mathrm{~B}_{\mathrm{g}}\right)$ & $156\left(\mathrm{~A}_{\mathrm{u}}\right)$ & $121\left(\mathrm{~B}_{\mathrm{g}}\right)$ & $80\left(\mathrm{~A}_{\mathrm{u}}\right)$ & $43\left(\mathrm{~B}_{\mathrm{g}}\right)$ & $18\left(\mathrm{~A}_{\mathrm{u}}\right)$ \\
\hline & & $\kappa$ & 0.083 & $0.167^{\circ}$ & 0.250 & 0.333 & 0.417 & 0.500 & 0.583 & 0.667 & 0.750 & $0.833^{\circ}$ & 0.917 \\
\hline & $\operatorname{sp}\left(C_{1}\right)$ & exptl (INS) & 115 & 140 & 163 & 190 & 208 & 216 & 190 & 170 & 140 & 128 & 96 \\
\hline & & & & & 170 & & 197 & 208 & & 163 & & & \\
\hline & & $\mathrm{av}^{b}$ & & & 167 & & 203 & 212 & & 167 & & & \\
\hline & & exptl (Ram) & 112 & 140 & & & 200 & & & & 140 & & \\
\hline & & calcd & 46 & 86 & 122 & 157 & 174 & 183 & 157 & 122 & 86 & 54 & 21 \\
\hline & & $\kappa$ & 0.083 & 0.167 & 0.250 & 0.333 & 0.417 & 0.500 & 0.583 & 0.667 & 0.750 & 0.833 & 0.917 \\
\hline
\end{tabular}

${ }^{a}$ At the B3LYP/6-31G* level. $i=1,2, \ldots,(N-3) ; \kappa=i /(N-2) ; N$ represents the number of beads in the chain, and $k$ is the corresponding wavevector. ${ }^{b}$ Mean value of the observed Davydov components.

same INS intensity. When comparing the experimental INS and Raman data (Figure 2), it is easily verified that while almost all of the TAMs are detected by INS, only some of these transverse modes are observed in the Raman spectra (Tables 1 
TABLE 2: Transverse Acoustic Vibrational Modes (TAMs) for the $\mathrm{D}_{2} \mathrm{~N}\left(\mathrm{CH}_{2}\right)_{n} \mathrm{ND}_{2}(n=2-10, N=12)$ Diamines, Spermidine- $N-d_{5}$ and Spermine- $N-d_{6}$

\begin{tabular}{|c|c|c|c|c|c|c|c|c|c|c|c|c|c|}
\hline \multirow[b]{2}{*}{$N$} & & & \multicolumn{11}{|c|}{$i$} \\
\hline & & & 1 & 2 & 3 & 4 & 5 & 6 & 7 & 8 & 9 & 10 & 11 \\
\hline \multirow[t]{3}{*}{4} & 1,2 dae $\left(C_{2 h}\right)$ & $\begin{array}{l}\text { exptl (INS) } \\
\text { exptl (Ram) }\end{array}$ & & & & & & & & & & & \\
\hline & & $\operatorname{calcd}^{a}$ & $132\left(A_{u}\right)$ & & & & & & & & & & \\
\hline & & $\kappa$ & 0.500 & & & & & & & & & & \\
\hline \multirow[t]{3}{*}{5} & 1,3dap $\left(C_{2 v}\right)$ & $\begin{array}{l}\text { exptl (INS) } \\
\text { av }^{b} \\
\text { exptl (Ram) }\end{array}$ & & & & & & & & & & & \\
\hline & & calcd & $112\left(\mathrm{~A}_{2}\right)$ & $112\left(\mathrm{~B}_{1}\right)$ & & & & & & & & & \\
\hline & & $\kappa$ & 0.333 & 0.666 & & & & & & & & & \\
\hline \multirow[t]{4}{*}{6} & $1,4 \mathrm{dab}\left(C_{2 h}\right)$ & $\begin{array}{l}\text { exptl (INS) } \\
\mathrm{av}^{b}\end{array}$ & 150 & 189 & 106 & & & & & & & & \\
\hline & & exptl (Ram) & 160 & & 110 & & & & & & & & \\
\hline & & calcd & $95\left(\mathrm{~A}_{\mathrm{u}}\right)$ & $159\left(\mathrm{~B}_{\mathrm{g}}\right)$ & $74\left(\mathrm{~A}_{\mathrm{u}}\right)$ & & & & & & & & \\
\hline & & $\kappa$ & 0.250 & 0.500 & 0.750 & & & & & & & & \\
\hline \multirow[t]{4}{*}{7} & 1,5 dap $\left(C_{2 v}\right)$ & $\begin{array}{l}\text { exptl (INS) } \\
\mathrm{av}^{b}\end{array}$ & & 183 & & & & & & & & & \\
\hline & & exptl (Ram) & & & 179 & & & & & & & & \\
\hline & & calcd & $80\left(\mathrm{~A}_{2}\right)$ & $153\left(\mathrm{~B}_{1}\right)$ & $147\left(\mathrm{~A}_{2}\right)$ & $66\left(B_{1}\right)$ & & & & & & & \\
\hline & & $\kappa$ & 0.200 & 0.400 & 0.600 & 0.800 & & & & & & & \\
\hline \multirow[t]{6}{*}{8} & 1,6 dah $\left(C_{2 h}\right)$ & exptl (INS) & 115 & 178 & 203 & 152 & 104 & & & & & & \\
\hline & & & & 173 & 194 & & & & & & & & \\
\hline & & $a v^{b}$ & & 176 & 199 & & & & & & & & \\
\hline & & exptl (Ram) & & 176 & & 147 & & & & & & & \\
\hline & & calcd & $64\left(\mathrm{~A}_{u}\right)$ & $134\left(\mathrm{~B}_{\mathrm{g}}\right)$ & $171\left(\mathrm{~A}_{\mathrm{u}}\right)$ & $116\left(\mathrm{~B}_{\mathrm{g}}\right)$ & $48\left(\mathrm{~A}_{\mathrm{u}}\right)$ & & & & & & \\
\hline & & $\kappa$ & 0.167 & 0.333 & 0.500 & 0.667 & 0.833 & & & & & & \\
\hline \multirow[t]{4}{*}{9} & $1,7 \mathrm{dah}\left(C_{2 v}\right)$ & $\begin{array}{l}\text { exptl (INS) } \\
\mathrm{av}^{b}\end{array}$ & 132 & 161 & 193 & 183 & 150 & & & & & & \\
\hline & & exptl (Ram) & & 161 & & & 146 & & & & & & \\
\hline & & calcd & $66\left(\mathrm{~A}_{2}\right)$ & $122\left(\mathrm{~B}_{1}\right)$ & $168\left(\mathrm{~A}_{2}\right)$ & $160\left(B_{1}\right)$ & $101\left(\mathrm{~A}_{2}\right)$ & $45\left(B_{1}\right)$ & & & & & \\
\hline & & $\kappa$ & 0.143 & 0.286 & 0.429 & 0.571 & 0.714 & 0.857 & & & & & \\
\hline \multirow[t]{10}{*}{10} & 1,8 dao $\left(C_{2 h}\right)$ & exptl (INS) & 112 & 155 & 185 & 204 & 168 & 138 & 102 & & & & \\
\hline & & & & & 198 & 198 & & & & & & & \\
\hline & & $a v^{b}$ & & & 192 & 201 & & & & & & & \\
\hline & & exptl (Ram) & 116 & 148 & & & 165 & 135 & & & & & \\
\hline & & calcd & $55\left(\mathrm{~A}_{\mathrm{u}}\right)$ & $107\left(\mathrm{~B}_{\mathrm{g}}\right)$ & $156\left(\mathrm{~A}_{\mathrm{u}}\right)$ & $170\left(\mathrm{~B}_{\mathrm{g}}\right)$ & $134\left(\mathrm{~A}_{\mathrm{u}}\right)$ & $80\left(B_{g}\right)$ & $32\left(\mathrm{~A}_{\mathrm{u}}\right)$ & & & & \\
\hline & & $\kappa$ & 0.125 & 0.250 & 0.375 & 0.500 & 0.625 & 0.750 & 0.875 & & & & \\
\hline & $\operatorname{spd}\left(C_{1}\right)$ & $\begin{array}{l}\text { exptl (INS) } \\
\mathrm{av}^{b}\end{array}$ & & 163 & 190 & 205 & 175 & 143 & 108 & & & & \\
\hline & & exptl (Ram) & 130 & & 190 & & 177 & 148 & 110 & & & & \\
\hline & & calcd & 61 & 108 & 159 & 174 & 134 & 83 & 33 & & & & \\
\hline & & $\kappa$ & 0.125 & 0.250 & 0.375 & 0.500 & 0.625 & 0.750 & 0.875 & & & & \\
\hline \multirow[t]{4}{*}{11} & $1,9 \mathrm{dan}\left(C_{2 v}\right)$ & $\begin{array}{l}\text { exptl (INS) } \\
\mathrm{av}^{b}\end{array}$ & & 154 & 185 & 201 & 196 & 168 & 135 & 104 & & & \\
\hline & & exptl (Ram) & 120 & & & & 199 & & & 105 & & & \\
\hline & & calcd & $58\left(\mathrm{~A}_{2}\right)$ & $102\left(B_{1}\right)$ & $144\left(\mathrm{~A}_{2}\right)$ & $175\left(\mathrm{~B}_{1}\right)$ & $169\left(\mathrm{~A}_{2}\right)$ & $124\left(\mathrm{~B}_{1}\right)$ & $73\left(\mathrm{~A}_{2}\right)$ & $33\left(\mathrm{~B}_{1}\right)$ & & & \\
\hline & & $\kappa$ & 0.111 & 0.222 & 0.333 & 0.444 & 0.556 & 0.667 & 0.778 & 0.889 & & & \\
\hline \multirow[t]{4}{*}{12} & $1,10 \mathrm{dad}\left(C_{2 h}\right)$ & $\begin{array}{l}\text { exptl (INS) } \\
\mathrm{av}^{b}\end{array}$ & 120 & 143 & 164 & 198 & 203 & 177 & 152 & 135 & 107 & & \\
\hline & & exptl (Ram) & & & & 197 & & 173 & & 132 & & & \\
\hline & & calcd & $53\left(\mathrm{~A}_{\mathrm{u}}\right)$ & $94\left(\mathrm{~B}_{\mathrm{g}}\right)$ & $134\left(\mathrm{~A}_{\mathrm{u}}\right)$ & $170\left(\mathrm{~B}_{\mathrm{g}}\right)$ & $177\left(\mathrm{~A}_{\mathrm{u}}\right)$ & $151\left(\mathrm{~B}_{\mathrm{g}}\right)$ & $108\left(\mathrm{~A}_{\mathrm{u}}\right)$ & $62\left(\mathrm{~B}_{\mathrm{g}}\right)$ & $27\left(\mathrm{~A}_{\mathrm{u}}\right)$ & & \\
\hline & & $\kappa$ & 0.100 & 0.200 & 0.300 & 0.400 & 0.500 & 0.600 & 0.700 & $0.800^{\circ}$ & 0.900 & & \\
\hline \multirow[t]{10}{*}{14} & $1,12 \mathrm{dad}\left(C_{2 h}\right)$ & $\begin{array}{l}\text { exptl (INS) } \\
\mathrm{av}^{b}\end{array}$ & 106 & 131 & 145 & 181 & 202 & 206 & 186 & 150 & 134 & 106 & 85 \\
\hline & & exptl (Ram) & & 131 & & 173 & & & & 149 & & 109 & \\
\hline & & calcd & $40\left(\mathrm{~A}_{\mathrm{u}}\right)$ & $76\left(\mathrm{~B}_{\mathrm{g}}\right)$ & $113\left(\mathrm{~A}_{\mathrm{u}}\right)$ & $146\left(\mathrm{~B}_{\mathrm{g}}\right)$ & $171\left(\mathrm{~A}_{\mathrm{u}}\right)$ & $171\left(\mathrm{~B}_{\mathrm{g}}\right)$ & $155\left(\mathrm{~A}_{\mathrm{u}}\right)$ & $119\left(\mathrm{~B}_{\mathrm{g}}\right)$ & $76\left(\mathrm{~A}_{\mathrm{u}}\right)$ & $44\left(\mathrm{~B}_{\mathrm{g}}\right)$ & $18\left(\mathrm{~A}_{\mathrm{u}}\right)$ \\
\hline & & $\kappa$ & 0.083 & $0.167^{\circ}$ & 0.250 & 0.333 & 0.417 & 0.500 & 0.583 & 0.667 & 0.750 & $0.833^{\circ}$ & 0.917 \\
\hline & $\operatorname{sp}\left(C_{1}\right)$ & exptl (INS) & 114 & 138 & 158 & 181 & 192 & 206 & 185 & 168 & 138 & 125 & 95 \\
\hline & & & & & & 185 & 206 & & 181 & & & & \\
\hline & & $a v^{b}$ & & & & 183 & 199 & & 183 & & & & \\
\hline & & exptl (Ram) & 111 & 132 & 156 & 179 & 190 & & 179 & & 132 & & \\
\hline & & calcd & 45 & 82 & 113 & 151 & 172 & 178 & 151 & 122 & 84 & 50 & 20 \\
\hline & & $\kappa$ & 0.083 & 0.167 & 0.250 & 0.333 & 0.417 & 0.500 & 0.583 & 0.667 & 0.750 & 0.833 & 0.917 \\
\hline
\end{tabular}

${ }^{a}$ At the B3LYP/6-31G* level. $i=1,2, \ldots,(N-3) ; \kappa=i /(N-2) ; N$ represents the number of beads in the chain, and $k$ is the corresponding wavevector. ${ }^{b}$ Mean value of the observed Davydov components.

and 2). In fact, some of the TAMs are either very weak or symmetry forbidden transitions in Raman spectroscopy. Nevertheless, these optical selection rules were not always obeyed (e.g., 1,4-diaminobutane, Tables 1 and 2), which can be explained by the significant mixing of these transverse vibrations with external modes (as discussed later). Deuteration of the samples led to the anticipated shifts to lower frequencies in both Raman and INS (Figure 3), as a result of the variations in both 


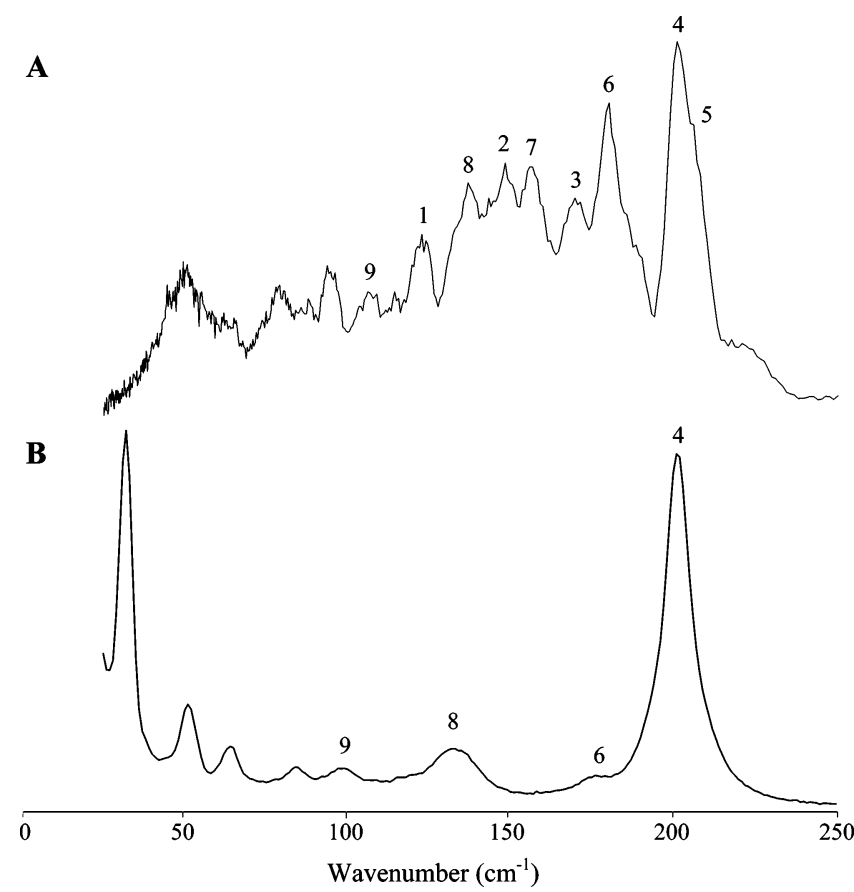

Figure 2. Experimental INS (A) and Raman (B) spectra (16-250 $\mathrm{cm}^{-1}$ ) for 1,10-diaminodecane. (The numbers refer to the corresponding TAM modes.)

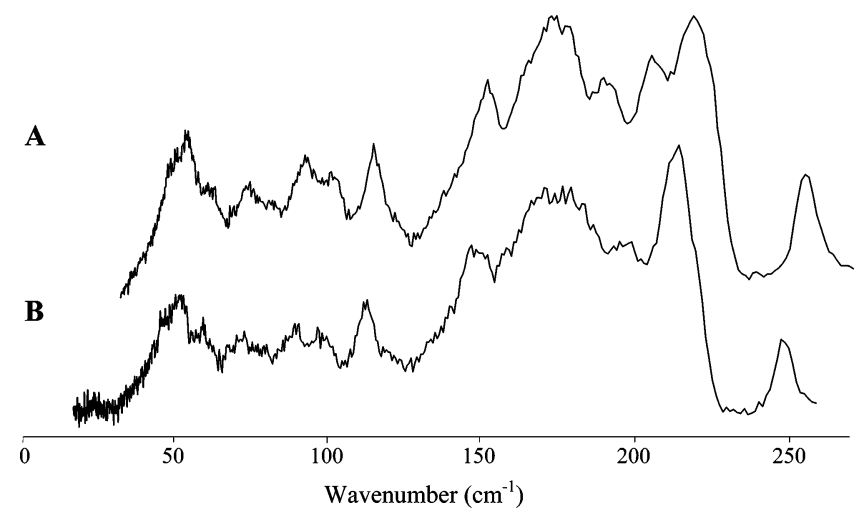

Figure 3. Experimental INS spectra $\left(16-260 \mathrm{~cm}^{-1}\right.$, at $\left.20 \mathrm{~K}\right)$ for spermidine, in its undeuterated (A) and $\mathrm{N}$-deuterated (B) forms.

mass (frequencies) and scattering cross section (INS intensities). This shift was found to be about $2-3 \mathrm{~cm}^{-1}$ for the TAM modes (Tables 1 and 2).

The transverse acoustic modes observed for the aliphatic amines studied here are directly related to their torsional vibrations, with each pair of carbons along the chain giving rise to an internal torsional coordinate, yielding a total of $N-3$ TAMs (Tables 1 and 2). These low frequency bands are prone to intermolecular interactions and are thus dependent on the molecular packing in the condensed phase. This explains why there are more transverse modes observed experimentally then predicted theoretically. Davydov splitting was detected for many of the TAMs in the INS spectra, probably due to the occurrence of distinct solid state phases as a result of the rapid cooling to the low temperatures used in INS, leading to two crystallographically and energetically inequivalent conformations. This doubling of the unit cell was previously observed for $n$-alkanes ${ }^{11}$ and extensively discussed for the particular case of $n$-octane, ${ }^{30}$ for which spectral evidence of a low temperature $(\sim 50 \mathrm{~K})$ phase transition was reported, suggesting that this may be common to all $n$-alkanes as well as to the structurally similar linear amines presently studied.
Furthermore, the presence of several molecules in a crystal (or a packed solid state) generates an external force field, which is responsible for new vibrational modes, the so-called external modes (we locate these below $\sim 120 \mathrm{~cm}^{-1}$ for the polyamines, as opposed to $80 \mathrm{~cm}^{-1}$ for the $n$-alkanes $\left.{ }^{11}\right)$. Those external modes, of appropriate character, mix with the TAMs, and the closer they are in frequency, the larger the extent of mixing. Thus, the lower frequency TAMs are more affected by this mixing process, while the high frequency ones (those with intermediate $\kappa$ values, see later) are less affected. As a result of this mode mixing, the experimental TAMs are pushed to higher wavenumbers than the external modes. We identify the gap detected around $120 \mathrm{~cm}^{-1}$ in the INS pattern of the polyamines, and in the large number of bands observed between $\sim 120$ and $210 \mathrm{~cm}^{-1}$ (Figure 4), with that expected between the internal and external modes. The fact that the external mode region is extended to higher frequencies for the linear amines than the $n$-alkanes is mainly due to the occurrence of intermolecular $\mathrm{R}-\mathrm{HN}-\mathrm{H} \cdots: \mathrm{NH}_{2}-\mathrm{R}$ contacts.

While the density of states corresponding to the external modes is not comprised of individual transitions, the observed TAMs remain sharp features. Although the impact of this mixing is more obvious for the TAM modes, similar effects were reported for some of the LAMs in these systems, ${ }^{10}$ namely, for the lowest frequency LAM1 of the longest amines. Also, a slight TAM-LAM coupling may be detected for wavenumbers near $200 \mathrm{~cm}^{-1}$; in fact, the LAM1 frequency was found to tend to the value of the presently reported TAM ${ }_{\text {max }}\left(\sim 209 \mathrm{~cm}^{-1}\right.$, Tables 1 and 2) upon lengthening of the chain. ${ }^{10}$

A very good agreement was found between the INS experimental TAMs presently observed and the low frequency neutron scattering pattern of the corresponding $n$-alkanes ${ }^{11}$ (Figure 5), which corroborates the idea of a significant conformational similarity (in the solid state) between these two sets of compounds. While the vibrational pattern of the $n$-alkanes comprises $\mathrm{CH}_{3}$ torsions $\left(\tau_{\mathrm{CH}_{3}}\right)$ as isolated and intense features around $250 \mathrm{~cm}^{-1}$, in the linear amines presently investigated, the torsional modes of the terminal $\mathrm{NH}_{2}$ groups $\left(\tau_{\mathrm{NH}_{2}}\right)$ are not clearly observed by INS above $210 \mathrm{~cm}^{-1}$, as would be expected from the theoretical calculations. This suggests the occurrence of $\mathrm{R}-\mathrm{N}-\mathrm{H} \cdots \mathrm{NH}_{2}-\mathrm{R}$ intermolecular interactions, as previously discussed. ${ }^{10}$ Actually, the vibrational consequences of the intermolecular hydrogen bonds occurring in this kind of system cannot be predicted by the isolated molecule approach used in the calculations presently carried out, that consider only internal forces. Therefore, some discrepancies between calculated and experimental data are to be expected for the oscillators involved in intermolecular close contacts. Moreover, the result of the overlay between the external and internal modes is not accounted for in this theoretical treatment, which explains the significant differences between the experimental and predicted lowest TAM values (Tables 1 and 2).

In the low frequency region presently analyzed, 1,2-diaminoethane, the shortest element of the series, was found to display exceptional behavior, similarly to what was previously reported for its LAM modes. ${ }^{10}$ This is proposed to be due to the occurrence of dimers in the solid state for this small molecule, upon formation of intermolecular $\mathrm{H}_{2} \mathrm{~N} \cdots \mathrm{H}$ interactions: $\mathrm{H}_{2} \mathrm{~N}\left(\mathrm{CH}_{2}\right)_{2}-$ $\mathrm{NH}_{2} \cdots \mathrm{NH}_{2}\left(\mathrm{CH}_{2}\right)_{2} \mathrm{NH}_{2}$ (comprising eight beads). Actually, the INS low frequency pattern observed for 1,2-diaminoethane displays a reasonable accord with that of 1,6-diaminohexane $(n=8)$ (Figure 4).

Figure 6 displays the plots of the INS band center positions of the experimental TAMs observed for the undeuterated and 
A
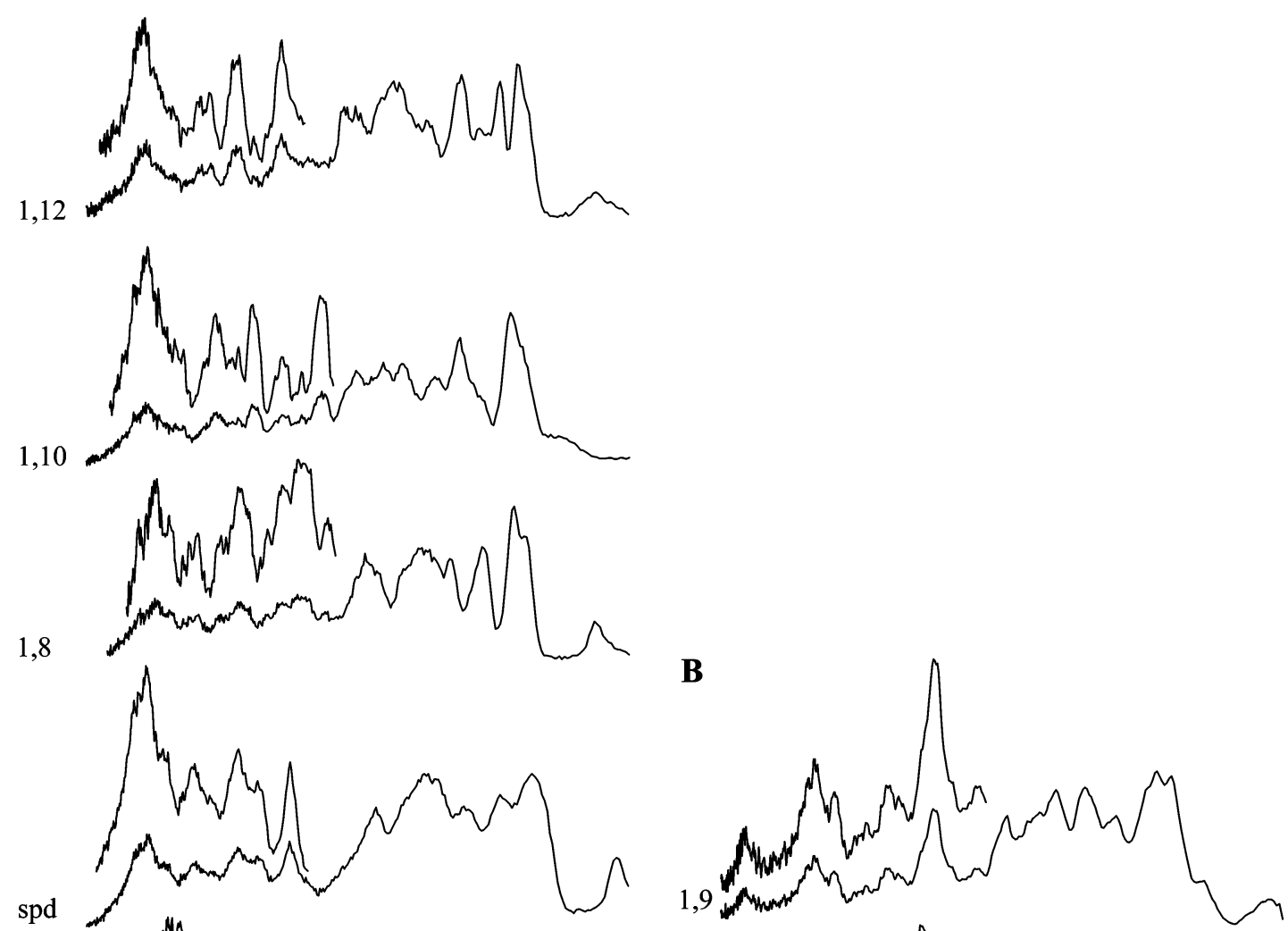

1,6

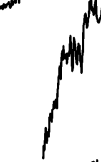

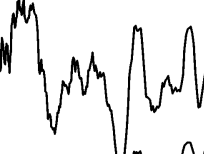

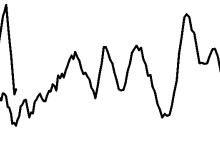
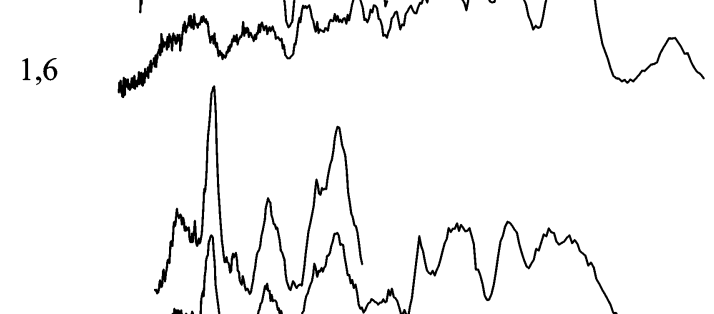

1,4

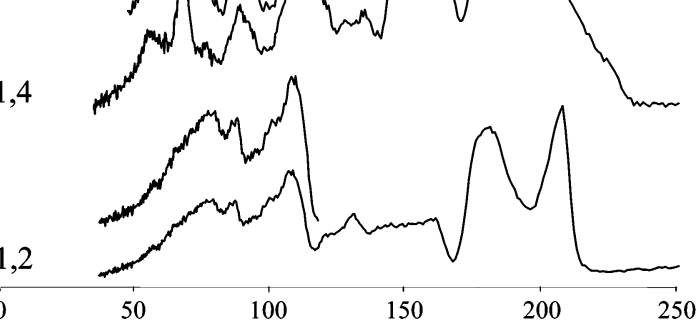

1,7

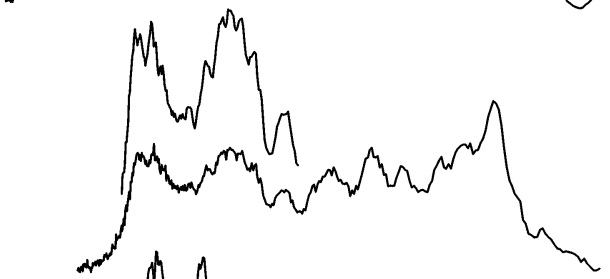

1,3

1,5

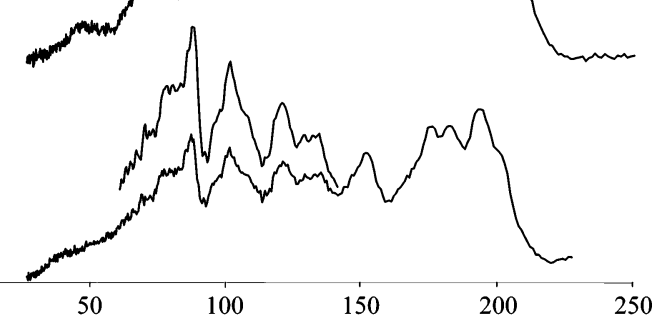

Wavenumber $\left(\mathrm{cm}^{-1}\right)$

Figure 4. Experimental INS spectra $\left(16-250 \mathrm{~cm}^{-1}\right.$, at $20 \mathrm{~K}$ ) for the series of polyamines studied: (A) $n=$ even; (B) $n=$ odd. (The following abreviations were used: $1, n=1,2-$ to $1,12-\mathrm{H}_{2} \mathrm{~N}\left(\mathrm{CH}_{2}\right)_{n} \mathrm{NH}_{2}(n=2-12)$; spd, spermidine; sp, spermine.)

deuterated amines, as a function of their ideal wavevectors $(\kappa)$. The upper frequency band-heads $\left(\left(\nu_{\mathrm{TAM}}\right)_{\max }\right)$ were observed at $\sim 212 \mathrm{~cm}^{-1}$ for the undeuterated molecules (Figure 6A) and at $206 \mathrm{~cm}^{-1}$ for the N-deuterated ones (Figure 6B). $\left(\nu_{\mathrm{TAM}}\right)_{\max }$ was found to display a steady increase as the amine chain lengthens, from 1,2-diaminoethane to 1,12-diaminododecane and spermine.
Moreover, the value of the ideal wavevectors $(\kappa)$ corresponding to a maximum TAM frequency, $\kappa_{\max }$, estimated to be 0.5 for both the undeuterated and $\mathrm{N}$-deuterated amines, remained nearly constant across the series. This is in accordance with what was previously verified for the LAM behavior of these same amines, ${ }^{10}$ as well as for the TAMs and LAMs of $n$-alkanes. ${ }^{9,11}$ 


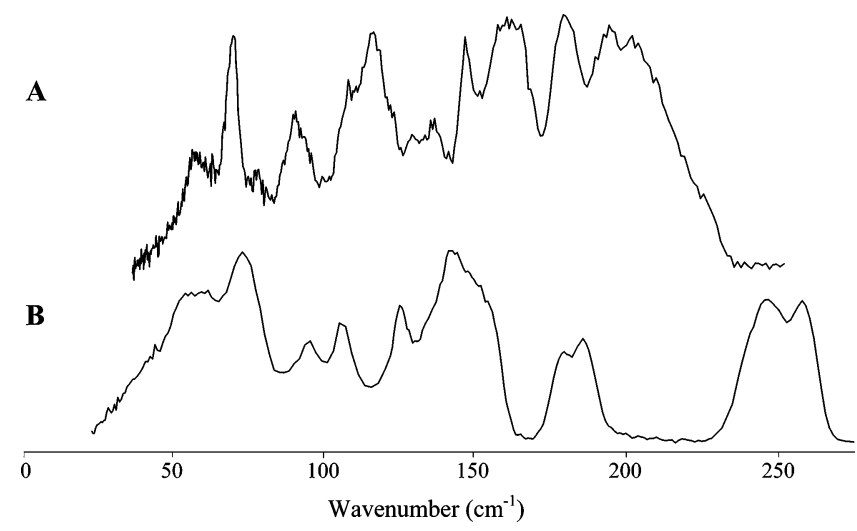

Figure 5. Experimental INS spectra $\left(16-275 \mathrm{~cm}^{-1}\right.$, at $\left.20 \mathrm{~K}\right)$ for $1,4-$ diaminobutane (A) and $n$-hexane (from ref 11) (B).
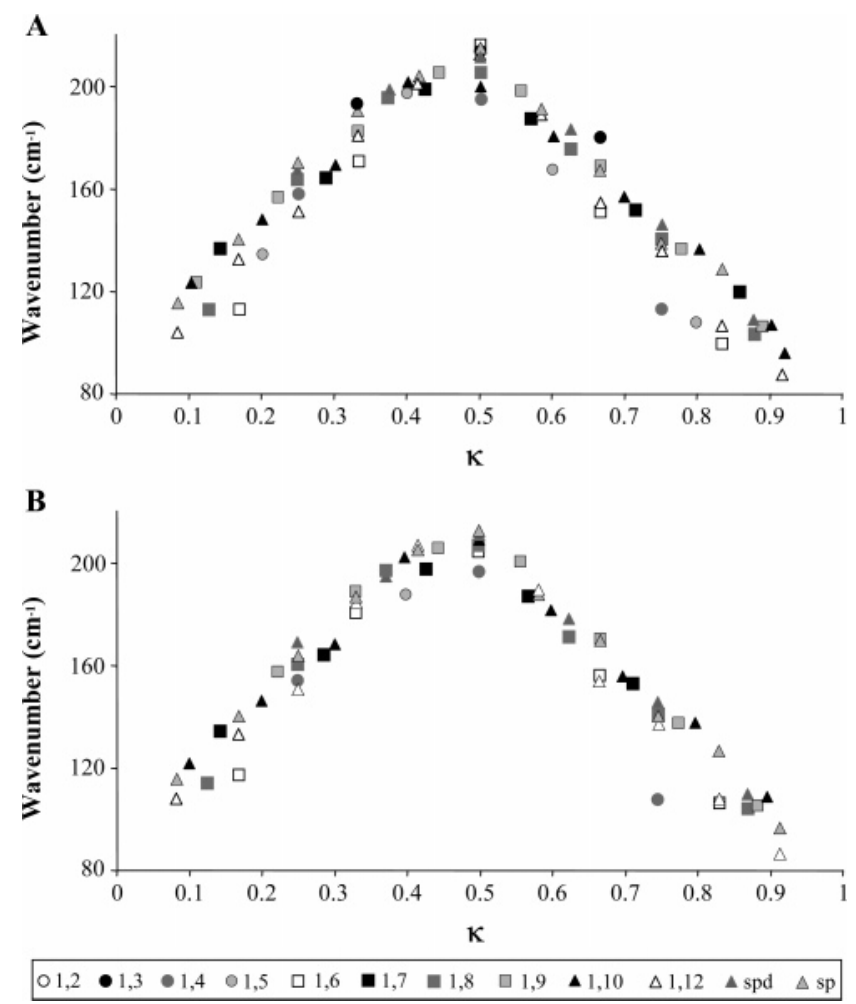

Figure 6. Plot of the INS band center positions $\left(\mathrm{cm}^{-1}\right)$ for the TAMs observed for the series of polyamines studied, as a function of their ideal wavevectors $(\kappa)$ : (A) undeuterated amines; (B) N-deuterated amines. $\left(\mathrm{TAM}_{\max } \sim 200 \mathrm{~cm}^{-1}\right.$. The effects of Davydov splitting were averaged out. The following abreviations were used: $1, n=1,2-$ to 1,12- $\mathrm{H}_{2} \mathrm{~N}\left(\mathrm{CH}_{2}\right)_{n} \mathrm{NH}_{2}(n=2-12)$; spd, spermidine; sp, spermine).

Since the observed $\kappa_{\max }$ did not deviate from the model value of 0.5 , it is suggested that the dynamic coupling of the chain torsional modes with the higher frequency $\mathrm{CH}_{2}$ rocking vibrations (between $\sim 800$ and $1250 \mathrm{~cm}^{-1}$ ) is not significant in these systems.

An $n$-even $/ n$-odd dependence of the low frequency INS vibrational spectra was found for the series of polyamines studied, in agreement with the reported results for their vibrational data in the region above $250 \mathrm{~cm}^{-1},{ }^{10}$ and also with the variations found for a homologous series of $n$-alkanes $(n=$ $5-25) .8,9,11$ This $n$-odd $/ n$-even alternation of physical properties and INS pattern should be related to a distinct packing arrangement of the linear amines, which is expected to be tighter for even-membered molecules and less dense for $n=$ odd, similarly to that was previously recognized for $n$-alkanes. ${ }^{31,32}$
Therefore, the TAM dispersion curves for the two sets of polyamines display only subtle differences.

\section{Conclusions}

The use of the complementary INS and Raman spectroscopic techniques, coupled to DFT calculations, allowed the complete assignment of the TAM modes for the series of linear aliphatic amines $\mathrm{H}_{2} \mathrm{~N}\left(\mathrm{CH}_{2}\right)_{n} \mathrm{NH}_{2}(n=2-10, n=12)$, spermidine and spermine, both in their undeuterated and $\mathrm{N}$-deuterated forms. The quantum mechanical calculations, performed at the B3LYP/6-31G* level for the all-trans conformations of the polyamines, were showed to adequately mimic these kinds of linear nitrogen-containing molecules.

An $n$-even/ $n$-odd dependence of the low frequency neutron scattering pattern was observed, probably as the result of a distinct packing arrangement of the amines in the condensed phase, namely, the tighter packing of the even-membered chains relative to the odd-membered ones (as previously reported for $n$-alkanes $\left.{ }^{11}\right)$.

The conformational similarity (in the solid state) between the linear aliphatic polyamines studied in this work and the analogous $n$-alkanes justifies the remarkably good accordance between the corresponding INS experimental TAMs (similarly to what was previously reported for the LAM modes ${ }^{8-10}$ ).

The present study, coupled to the previously reported work on the same polyamine series, ${ }^{10}$ has allowed the achievement of a complete and reliable assignment of all the low energy modes of these systems, both tranverse and longitudinal. Knowledge of the conformational behavior of linear polyamines, strongly determined by intermolecular $\mathrm{R}-\mathrm{HN}-\mathrm{H} \cdots \mathrm{NH}_{2}-\mathrm{R}$ hydrogen-type interactions mainly ocurring in condensed phases, may thus help one to better understand the diverse and often vital biological role of these kinds of molecules (namely, in cell growth and differentiation processes and in cancer prevention and therapy).

Acknowledgment. Financial support from the Portuguese Foundation for Science and Technology is acknowledged, project POCTI/47256/QUI/2002 (cofinanced by the European Community fund FEDER).

\section{References and Notes}

(1) Tabor, C. W.; Tabor, H. Annu. Rev. Biochem. 1984, 53749 and references therein.

(2) Auvinen, M.; Passinen, A.; Anderson, L. C.; Holtta, E. Nature (London) 1992, 360, 355

(3) O'Brien, T. G.; Megosh, L. C.; Gilliard, G.; Soler, A. P. Cancer Res. 1997, 57, 2630 and references therein.

(4) Xie, X.; Gillies, R. J.; Gerner, E. W. J. Biol. Chem. 1997, 272, 20484.

(5) Vassileva, V.; Ignatov, G. Bulg. J. Plant Physiol. 1999, 25, 49.

(6) Guo, D.; Lu, Z. J. Gen. Physiol. 2000, 115, 783.

(7) Medina, M. A.; Urdiales, J. L.; Rodriguez-Caso, C.; Ramírez, F. J.; Sánchez-Juménez, F. Crit. Rev. Biochem. Mol. Biol. 2003, 38, 23.

(8) Parker, S. F.; Braden, D. A.; Tomkinson, J.; Hudson, B. S. J. Phys. Chem. B 1998, 102, 5955.

(9) Braden, D. A.; Parker, S. F.; Tomkinson, J.; Hudson, B. S. J. Chem. Phys. 1999, 111, 429.

(10) Marques, M. P. M.; Batista de Carvalho, L. A. E.; Tomkinson, J. J. Phys. Chem. A 2002, 106, 2473.

(11) Tomkinson, J.; D. A.; Parker, S. F.; Braden, D. A.; Hudson, B. S. Phys. Chem. Chem. Phys. 2002, 4, 716.

(12) www.isis.rl.ac.uk.

(13) Frisch, M. J.; et al. Gaussian 98, revision A.9; Gaussian Inc.: Pittsburgh, PA, 1998.

(14) Lee, C.; Yang, W.; Parr, R. G. Phys. Rev. 1988, B37, 785.

(15) Miehlich, B.; Savin, A.; Stoll, H.; Preuss, H. Chem. Phys. Lett. 1989, 157, 200

(16) Becke, A. D. Phys. Rev. 1988, A38, 3098.

(17) Becke, A. D. J. Chem. Phys. 1993, 98, 5648. 
(18) Hariharan, P. C.; Pople, J. A. Theor. Chim. Acta 1973, 28, 213.

(19) Peng, C.; Ayala, P. Y.; Schlegel, H. B.; Frisch, M. J. J. Comput. Chem. 1996, 17, 49.

(20) Ramirez-Cuesta, A. J. Comput. Phys. Commun. 2004, 157 (3), 226

(21) Batista de Carvalho, L. A. E.; Amorim da Costa, A. M.; TeixeiraDias, J. J. C. THEOCHEM 1990, 205, 327.

(22) Batista de Carvalho, L. A. E.; Lourenço, L. E.; Marques, M. P. M. J. Mol. Struct. 1999, 482-483, 639 and references therein.

(23) Marques, M. P. M.; Batista de Carvalho, L. A. E. In COST 917: Biogenically Active Amines in Food; Morgan, D. M. L., White, A., SánchezJiménez, F., Bardocz, S., Eds.; European Comission: Luxemburg, 2000; Vol. IV, p 122.

(24) Mirkin, N. G.; Krimm, S. J. Phys. Chem. 1993, 97, 13887 and references therein.
(25) Giglio, E.; Liquori, A. M.; Puliti, R.; Ripamonti, A. Acta Crystallogr. 1966, 20, 652; 683.

(26) Taylor, R.; Kennard, O. J. Am. Chem. Soc. 1982, 104, 5063.

(27) Steiner, T. Chem. Commun. 1997, 727.

(28) Katrusiak, A. J. Mol. Struct. 1999, 474, 125.

(29) Desiraju, G. R.; Steiner, T. The Weak Hydrogen Bond in Structural Chemistry and Biology. IUCr Monographs on Chystallography-9; Oxford University Press: Oxford, U.K., 1999; see also references therein.

(30) Neumann, M. A.; Johnson, M. R.; Radaelli, P. G. Chem. Phys. 2001, 266, 53.

(31) Mathisen, H.; Norman, N.; Pedersen, B. F. Acta Chim. Scand. 1967, 21,127

(32) Barnes, J.; Franconi, B. J. Phys. Chem. Ref. Data 1978, 7, 1309 and references therein. 